\title{
Caracterização do ceceio em pacientes de um Centro Clínico de Fonoaudiologia
}

\section{Lisp characterization of patients from the Centro Clínico de Fonoaudiologia}

\author{
Antonelle Ferreira Leite ${ }^{1}$, Sabrina Barros Silva ${ }^{2}$, Ana Teresa Brandão de Oliveira e Britto ${ }^{3}$, \\ Camila Queiroz de Moraes Silveira Di Ninno ${ }^{4}$
}

\begin{abstract}
RESUMO
Objetivo: Este estudo pretendeu verificar e caracterizar a ocorrência de ceceio, investigando a correlação entre o ceceio e a tonicidade da sílaba com [s] em diferentes contextos silábicos, observar se alguns desses contextos facilitam a produção correta de [s] e devem ser indicados como recurso terapêutico. Métodos: Dos 54 pacientes em tratamento na clínica de Motricidade Oral do Centro Clínico de Fonoaudiologia foram selecionados, por meio da análise de prontuários, 16 pacientes na faixa etária entre 5 e 19 anos, de ambos os sexos e que apresentavam ceceio. Estes foram submetidos a teste de fala constituído da emissão de 79 palavras com [s] nas posições iniciais, finais e dentro da palavra, em sílabas átonas e tônicas seguidas das vogais [a], [ع], [e], [i], [פ], [o] e [u], registrado por meio de filmagem e gravação. Para análise estatística dos dados, foi utilizado o Teste Z para comparação de proporções e o software Minitab versão 14.0. Resultados: Doze (75\%) sujeitos pesquisados apresentaram ceceio anterior e 4 (25\%), ceceio lateral. Não se observou diminuição do ceceio em relação à tonicidade da sílaba ou à co-articulação. Houve diminuição significativa do ceceio nas posições ataque inicial e ataque medial em relação às posições coda medial e final. Conclusão: A tonicidade silábica e a co-articulação com as diferentes vogais não alteram a incidência do ceceio. As posições iniciais de sílaba, porém, parecem ser facilitadoras da produção de [s] e devem, portanto ser consideradas na escolha do recurso e material a ser utilizado em terapia.
\end{abstract}

DESCRITORES: Distúrbios de fala/etiologia; Transtornos da articulação; Fonética

\section{INTRODUÇÃO}

A fala é um meio de comunicação característico do ser humano, que abre possibilidades de experiências educacionais e sociais, contribuindo para o bem estar e a saúde do homem. Nela estão envolvidos articuladores móveis e articuladores fixos, sendo esta a atividade motora mais rápida e complexa do ser humano e a que envolve maior número de fibras motoras ${ }^{(1)}$.

Trabalho realizado na Pontifícia Universidade Católica de Minas Gerais PUC-Minas - Belo Horizonte (MG), Brasil.

(1) Especializanda em Motricidade Oral pela Pontifícia Universidade Católica de Minas Gerais - PUC-Minas - Belo Horizonte (MG), Brasil.

(2) Especializanda em Motricidade Oral pela Pontifícia Universidade Católica de Minas Gerais - PUC-Minas - Belo Horizonte (MG), Brasil.

(3) Pós-graduanda em Lingüística pela Pontifícia Universidade Católica de Minas Gerais - PUC-Minas - Belo Horizonte (MG), Brasil; Professora Assistente do Curso de Fonoaudiologia e do Curso de Especialização em Motricidade Orofacial da Pontifícia Universidade Católica de Minas Gerais - PUC-Minas - Belo Horizonte (MG), Brasil.

(4) Pós-graduanda em Lingüística pela Universidade Federal de Minas Gerais - UFMG - Belo Horizonte (MG), Brasil; Professora Assistente do Curso de Fonoaudiologia e do Curso de Especialização em Motricidade Orofacial da Pontifícia Universidade Católica de Minas Gerais - PUC-Minas - Belo Horizonte (MG), Brasil.

Endereço para correspondência: Antonelle Ferreira Leite. R. Sandoval Campos, 27, Bairro Álvaro Camargo, Belo Horizonte - MG, CEP 30860-100.

E-mail: antonelleleite@yahoo.com.br

Recebido em: 21/11/2007; Aceito em: 28/2/2008
A fim de se expressar verbalmente e de forma inteligível, é necessário desenvolver tanto o aspecto fonético (produção) quanto o aspecto fonológico (organização) do sistema de fala ${ }^{(2-3)}$. A fala envolve vários aspectos lingüísticos, motores, cognitivos e orgânicos, adquiridos e organizados no período entre dois e oito anos de idade ${ }^{(4-5)}$.

Alterações na aquisição fonética e fonológica podem gerar desvios de fala. $\mathrm{O}$ desvio fonético tem como principais causas as alterações de estruturas ósseas e/ou musculares, envolvidas na articulação e nas alterações de produção da fala; o desvio fonológico, por sua vez, ocorre na ausência de alterações orgânicas originando-se nas dificuldades de contextualização dos sons, na organização lingüística e na combinação dos traços fonêmicos ${ }^{(2,6)}$. As alterações fonéticas e fonológicas devem receber diagnóstico diferenciado, uma vez que o tratamento destes quadros passa por procedimentos terapêuticos distintos. Basicamente, o critério de distinção entre essas alterações é o estabelecimento ou não de contraste entre os sons da fala. Nas alterações fonológicas, observa-se a eliminação do contraste entre os sons ocasionando omissões e substituições fonêmicas ${ }^{(7)}$. As alterações fonéticas ou distorções fonêmicas podem ser definidas como a substituição de um som padrão por um som não padrão, mantendo o contraste entre o som distorcido e os demais sons da língua. 
Modificações estruturais da cavidade oral, tais como atresia do arco superior, má oclusão, face muito curta ou outras alterações decorrentes de traumas ou cirurgias alteram significativamente os pontos articulatórios levando a distorções ou imprecisões na fala, principalmente dos fonemas fricativos ${ }^{(3,8)}$

O equilíbrio das estruturas estomatognáticas ou orofaciais como dentes, lábios e língua, é importante na articulação de consoantes, pela alteração que provocam no fluxo de ar. Os fricativos são os sons mais fáceis de serem distorcidos por serem contínuos e, portanto, qualquer alteração no padrão de pressão do ar pela constrição anterior da cavidade oral pode resultar na distorção desse som ${ }^{(9)}$.

Para alguns pesquisadores, as irregularidades maxilo-mandibulares e dentárias (má-oclusão) prejudicam a realização de determinados fonemas, tornando-os distorcidos. Porém, devido às diferenças de metodologia, populações, variações lingüísticas e vários outros fatores, esses estudos não são considerados conclusivos ou não evidenciaram essa relação ${ }^{(9-10)}$.

Os fonemas fricativos línguo-alveolares /s/ e /z/ são produzidos com constrição da corrente aérea entre a ponta da língua e o alvéolo inferior. É necessário que a borda da língua assuma forma e posição específica e precisa para que o fluxo aéreo possa ser direcionado para os incisivos inferiores. Quando ocorre alteração dessas condições o som é produzido de forma incorreta $^{(11)}$. O termo ceceio se refere à incorreta produção dos fricativos línguo-alveolares. São vários os tipos de ceceio observados, dentre eles, os mais comuns são ${ }^{(7,12-13)}$ :

Ceceio central ou anterior: ocorre quando o sopro respiratório é emitido centralmente, mas a língua encontra-se mal posicionada nos planos vertical e ântero-posterior, em geral entre os dentes anteriores.

Ceceio lateral: ocorre quando a ponta da língua faz pressão sobre os incisivos centrais superiores ou no alvéolo, a corrente de ar é dividida e observa-se um escape lateral do ar pela cavidade oral.

Ceceio nasal: é relativamente raro, sendo decorrente de um insuficiente fechamento velofaríngeo, associado à retração da língua ${ }^{(7,12)}$.

O estudo da distorção fonética do /s/ é bastante complexo, uma vez que requer observações de diversos parâmetros fonéticos, oromiofuncionais, ambientais, e prosódicos ${ }^{(13)}$. A produção do fonema /s/, segundo estudos de análise acústica da fala, estaria estabilizada aos nove anos e seis meses de idade $^{(14)}$. Outros estudos afirmam que as crianças completam a aquisição dos fonemas com, no máximo, sete anos, mas que o controle motor de fala pode necessitar de um tempo mais longo, por volta dos 11 aos 13 anos de idade ${ }^{(11)}$. Os desvios quando persistentes na idade adulta, são na maioria de ordem fonética e de terapêutica mais complicada ${ }^{(15)}$.

Um estudo em crianças de três a seis anos mostrou associação entre a presença de ceceio e alteração oclusal (sendo a mordida aberta anterior isolada, o fator de maior ocorrência), e também evidenciou maior ocorrência em indivíduos do sexo feminino ${ }^{(9,16-17)}$. Na mordida aberta anterior, as bordas incisais dos dentes anteriores possuem um distanciamento que leva à modificação na posição da língua e, conseqüentemente, alteração na fricção do [s]. A mordida aberta anterior pode levar à elevação, protrusão ou interposição lingual como forma adaptativa de efetivar a produção desse fonema ${ }^{(16-17)}$. $\mathrm{Na}$ mordida aberta lateral, ocorre escape de ar lateral porque não há encontro das cúspides dos dentes molares superiores e inferiores, prejudicando a emissão do fonema /s/, levando a um som sibilante e/ou chiante. A mordida cruzada também pode interferir na produção correta desse fonema ${ }^{(7,11)}$.

Alguns autores salientam a interferência da tonicidade e da co-articulação na produção de diferentes fonemas. Um aspecto relevante em nossa pesquisa diz respeito à acentuação dos vocábulos. $\mathrm{O}$ acento em português é distintivo. Apresenta-se como uma maior força expiratória ou intensidade de emissão da vogal de uma sílaba, em contraste com as demais vogais silábicas ${ }^{(18)}$.

A importância deste trabalho está no fato da Fonoaudiologia carecer de estudos mais profundos sobre o sistema de sons do português brasileiro, fator agravante para a compreensão das alterações fonéticas e fonológicas dessa língua. É preciso conhecer melhor o contexto em que tais alterações ocorrem para melhor diagnosticá-las e tratá-las. Deve-se considerar que as dificuldades na articulação das palavras podem ter relação com a tonicidade silábica e com as vogais com as quais os sons co-articulam, podendo as mesmas ser facilitadoras ou não, na produção adequada do som.

Os objetivos deste estudo foram verificar e caracterizar a ocorrência do ceceio no fonema /s/; investigar a existência de correlação entre o ceceio e a tonicidade da sílaba, a coarticulação com as diferentes vogais e as diferentes posições na palavra; observar se alguns desses contextos facilitam a produção correta de /s/ e se poderiam ser indicados como recurso terapêutico.

\section{MÉTODOS}

A pesquisa foi submetida e aprovada pelo Comitê de Ética e Pesquisa da PUC Minas sob o número CAAE 0035.0213.00005 e considerada sem risco.

Dos 54 pacientes em tratamento fonoaudiológico, no setor de Motricidade Oral do Centro Clínico de Fonoaudiologia da PUC Minas, foram selecionados para este estudo 16 pacientes, na faixa etária de cinco a 19 anos de idade, de ambos os sexos, por apresentarem ceceio no fonema /s/. Os pacientes que não apresentavam ceceio foram excluídos da pesquisa.

Foi realizado um levantamento de dados dos protocolos de anamnese e de avaliação da motricidade oral dos prontuários dos 16 participantes da pesquisa. Os dados coletados foram referentes à idade, ao tempo de terapia, ao tipo de ceceio e ao tipo de mordida.

Os pacientes foram fotografados de frente e de perfil para registro de sua tipologia facial, com câmera fotográfica digital de 5.0 Mega Pixels, marca Canon ${ }^{\circledR}$.

$\mathrm{Na}$ avaliação de fala dirigida, foi solicitado ao paciente que nomeasse figuras isoladas, com o fonema /s/. Para tanto, foi elaborada, pelas autoras da pesquisa, uma lista de palavras com /s/ seguido de diferentes vogais, em sílabas tônicas e átonas e, nas posições ataque (onset) inicial (início de sílaba e início de palavra), ataque (onset) medial (início de sílaba dentro de palavra), coda medial (final de sílaba e dentro de palavra), coda final (final de sílaba e final de palavra). A lista 
de palavras (Anexo 1) foi utilizada com o objetivo de avaliar a presença ou não de ceceio, assim como classificar o tipo de ceceio encontrado. Os pacientes foram orientados a nomear as figuras, espontaneamente. Quando o paciente não fazia a nomeação espontaneamente, eram oferecidas pistas para sua nomeação. Caso ele ainda não conseguisse nomeá-la, era solicitada a repetição da palavra. A coleta dos dados foi feita conjuntamente por duas examinadoras, autoras da pesquisa.

Foi realizada ainda uma avaliação de fala espontânea por meio de conversa informal e de respostas a um roteiro prévio (Anexo 2).

Os procedimentos de coleta de dados de fala foram filmados com filmadora $\mathrm{JVC}^{\circledR}$, para registro e confronto posterior, caso houvesse necessidade.

Para análise da distorção fonêmica, foram utilizadas observações perceptivo-auditivas e visuais da produção articulatória dos sujeitos. O resultado da presença ou não de ceceio e do tipo do mesmo foi considerado a partir da concordância de ambas examinadoras, no momento da avaliação ou em posterior revisão das avaliações registradas nas filmagens.

O tratamento estatístico utilizado seguiu a metodologia descritiva, com a descrição das porcentagens dos participantes, com diminuição do ceceio quando a produção do fonema /s/ estava associada à tonicidade silábica, à posição do fonema na palavra e à co-articulação. Para a análise dos dados, foi considerada a produção correta ou incorreta do fonema /s/, ou seja, ausência ou presença do ceceio, para posteriormente relacioná-la à tonicidade e à co-articulação.

Foi aplicado o teste estatístico Z para comparação de proporções, visando a identificar uma possível diferença entre a tonicidade, a posição do fonema na palavra e à co-articulação na produção adequada do fonema /s/. Para averiguação das suposições, foi adotado o nível de significância de $5 \%(\mathrm{p}<0,05)$ para todos os testes estatísticos. Foi utilizado o software Minitab versão 14.0, para a obtenção dos resultados.

\section{RESULTADOS}

Dos 16 pacientes avaliados, $11(68,75 \%)$ encontram-se na faixa etária dos cinco aos 13 anos de idade e cinco $(31,25 \%)$ encontram-se na faixa etária entre 14 e 19 anos de idade, com média de idade de 11,5 anos e de ambos os sexos.

O tempo de terapia destes pacientes também variou de um mês a 21 meses de terapia fonoaudiológica, no Centro Clínico de Fonoaudiologia, com tempo médio de duração de 7,25 meses. Dos 16 pacientes, oito (50\%) tinham no máximo seis meses de terapia e os outros oito (50\%) tinham tempo de terapia superior a seis meses.

A Tabela 1 mostra os resultados relacionados à presença/ausência do ceceio de acordo com o posicionamento da fricativa na sílaba: observou-se diminuição significativa do ceceio quando o/s/ encontrava-se em posição ataque inicial e medial, quando comparado ao /s/ em posição coda medial e final $(\mathrm{p}=0,000)$.

Verifica-se nas Tabelas 2 e 3 que a tonicidade, independentemente da posição que o fonema ocupe na palavra, não interfere significativamente na produção do fonema /s/, não havendo diferença na produção correta/incorreta (valores de $p>0,05$ ).

Nas Tabelas 4 e 5 não se observou diminuição do ceceio quando a produção do fonema/s/ estava associada à co-articulação das vogais [a], [ع], [e], [i], [Э], [o] ou [u] (valores de $\mathrm{p}>0,05$ ).

A alteração de mordida encontrada com maior frequiência nos pacientes avaliados foi a mordida aberta anterior, correspondendo a uma porcentagem de 50\% dos casos (Figura 1).

Tabela 1. Resultados percentuais da relação posição do fonema /s/ na palavra e ocorrência de ceceio

\begin{tabular}{lcccc}
\hline Posicionamento & $N^{\circ}$ total palavras ditas & $N^{\circ}$ palavras distorcidas & $\%$ & Valor $p$ \\
\hline AI X AM & $464 / 224$ & $205 / 106$ & $0,441810 / 0473214$ & 0,439 \\
CM X CF & $352 / 224$ & $201 / 119$ & $0,571023 / 0537250$ & 0,350 \\
Al + AM X CM + CF & $688 / 576$ & $311 / 320$ & $0,452035 / 0,555556$ & 0,000 \\
\hline
\end{tabular}

Legenda: Al: ataque inicial; AM: ataque medial; CM: coda medial; CF: coda final

Teste estatístico Z

Tabela 2. Resultados percentuais da relação Tonicidade e Posição do fonema /s/ na palavra e sua produção distorcida (ceceio)

\begin{tabular}{lcccc}
\hline Tonicidade e Posição & $N^{\circ}$ total palavras ditas & $N^{\circ}$ palavras distorcidas & $\%$ & Valor $p$ \\
\hline TX A em Al & $224 / 240$ & $96 / 109$ & $0,428571 / 0,45167$ & 0,579 \\
TX A em AM & $112 / 112$ & $57 / 49$ & $0,508929 / 0,437500$ & 0,283 \\
TX A em CM & $176 / 176$ & $95 / 106$ & $0,539773 / 0,602273$ & 0,235 \\
TX A em CF & $96 / 28128$ & $50 / 69$ & $0,520833 / 0,539063$ & 0,787 \\
TX A em (Al+AM) & $336 / 352$ & $153 / 158$ & $0,455357 / 0,448864$ & 0,864 \\
TX A em (CM+CF) & $272 / 304$ & $145 / 175$ & $0,533088 / 0,575658$ & 0,304 \\
\hline
\end{tabular}

Legenda: Al: ataque inicial; AM: ataque medial; CM: coda medial; CF: coda final; T: tônica; A: átona

Teste estatístico Z

Tabela 3. Resultados percentuais da relação Tonicidade do fonema /s/ na palavra e sua produção distorcida (ceceio)

\begin{tabular}{lcccc}
\hline Tonicidade & $N^{\circ}$ total palavras ditas & $N^{\circ}$ palavras distorcidas & $\%$ & Valor $p$ \\
\hline Tônica $x$ Átona & $608 / 656$ & $298 / 333$ & $0,490132 / 0,507622$ & 0,534 \\
\hline
\end{tabular}

Legenda: $\mathrm{T}$ : tônica; $\mathrm{A}$ : átona

Teste estatístico $Z$ 
Tabela 4. Resultados percentuais da relação das Vogais associadas ao fonema /s/ na palavra e sua produção distorcida (ceceio)

\begin{tabular}{lcccc}
\hline Coarticulação & $N^{\circ}$ total palavras ditas & $N^{\circ}$ palavras distorcidas & $\%$ & Valor $p$ \\
\hline Somatório das Vogais T X A & $608 / 656$ & $300 / 322$ & $0,493421 / 0,490854$ & 0,927 \\
\hline
\end{tabular}

Legenda: T: tônica; A: átona; Teste estatístico Z

Tabela 5. Resultados percentuais da relação da Coarticulação /a/ associadas ao fonema /s/ na palavra e sua produção distorcida (ceceio)

\begin{tabular}{lcccc}
\hline Coarticulação /a/ & $N^{\circ}$ total palavras ditas & $N^{\circ}$ palavras distorcidas & $\%$ & Valor $p$ \\
\hline Somatório de T x A & $144 / 208$ & $74 / 97$ & $0,513889 / 0,466346$ & 0,380 \\
T x A em Al + AM & $96 / 192$ & $45 / 88$ & $0,468750 / 0,458333$ & 0,867 \\
T x A em CM + CF & $48 / 16$ & $29 / 09$ & $0,604167 / 0,562500$ & 0,770 \\
T (Al) x A (AM) & $208 / 80$ & $96 / 37$ & $0,461538 / 0,462500$ & 0,988 \\
T (CM) X A (CF) & $32 / 32$ & $20 / 18$ & $0,625000 / 0,562500$ & 0,610 \\
\hline
\end{tabular}

Legenda: Al: ataque inicial; AM: ataque medial; CM: coda medial; CF: coda final; T: tônica; A: átona

Teste estatístico Z

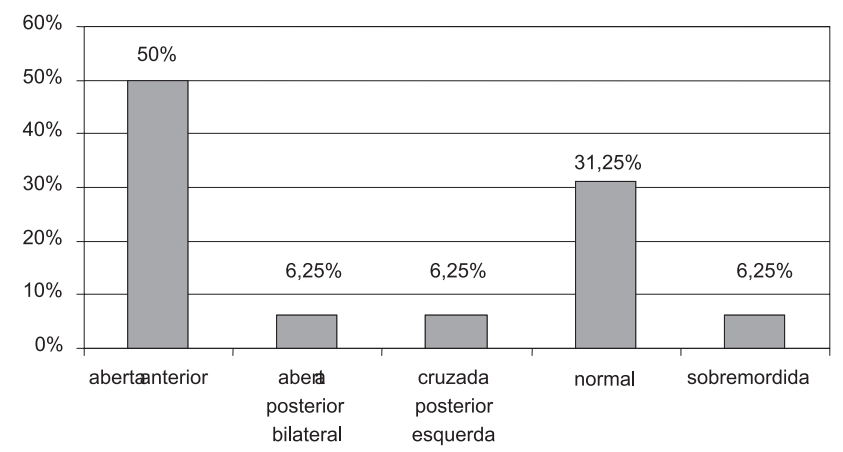

Figura 1. Distribuição de acordo com o tipo de mordida

A Figura 2 mostra a incidência do ceceio anterior totalizando $75 \%$ dos casos da amostra.

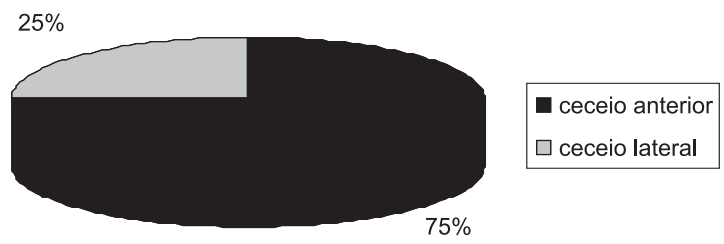

Figura 2. Distribuição de acordo com o tipo de ceceio

\section{DISCUSSÃO}

De acordo com os achados do presente estudo, observa-se não haver relação estatisticamente significante entre a ocorrência do ceceio e a tonicidade da sílaba.

Da mesma forma, não foi constatada relação significativa entre ceceio e co-articulação com as vogais [a], [ع], [e], [i], [פ], [o] e [u], não havendo variação na associação com as diferentes vogais.

Entretanto, quando se observam os achados relacionados ao posicionamento de /s/ na palavra, verifica-se que houve diminuição significativa do ceceio nas posições ataque inicial e medial da palavra em relação às posições de coda medial e final. O estudo considerou apenas a posição do fonema /s/ em sílaba tônica ou átona, sem levar em consideração se o fonema /s/ em silaba átona era pré-tônico ou pós-tônico.

Alguns autores salientam a interferência da tonicidade e da co-articulação na produção correta do fonema // $/{ }^{(19-20)}$, mas a análise estatística dos resultados encontrados no presente estudo contradiz estes dados, sugerindo que a incidência do ceceio não varia em função da tonicidade ou da co-articulação com diferentes vogais.

$\mathrm{Na}$ literatura pesquisada, alguns autores ${ }^{(19-20)}$ expõem a grande relação existente entre a produção do /s/ e o local de posicionamento na palavra. Estes enfatizam a incidência da diminuição do ceceio quando /s/ encontra-se nas posições iniciais da palavra, o que também foi verificado em nossos achados.

Deve-se salientar a importância e a possibilidade de algumas variáveis que não foram controladas, terem interferido no resultado da pesquisa, tais como o tempo de terapia, a diferença de idade entre os pacientes da pesquisa que, conseqüentemente, encontravam-se em estágios de aquisição fonológica também diferentes.

Os achados relacionados ao tempo de terapia mostram que oito (50\%) dos pacientes encontravam-se em terapia com tempo igual ou no máximo de seis meses de tratamento fonoaudiológico e oito (50\%) dos pacientes encontravam-se com tempo mínimo de seis meses e máximo de 21 meses de terapia fonoaudiológica. Não encontramos na literatura nenhum dado referente ao tempo de terapia, relacionado à presença/ausência do ceceio.

Com relação à idade, estudos afirmam que as crianças completam a aquisição dos fonemas com, no máximo, sete anos de idade, mas que o controle motor de fala pode necessitar de um tempo mais longo, até por volta dos 11 aos 13 anos de idade $^{(11)}$. Os achados da nossa pesquisa estão de acordo com a literatura, pois foi encontrado um número maior de pacientes com ceceio na faixa etária inferior aos 13 anos de idade. Os desvios, quando persistentes na idade adulta, são na maioria de ordem fonética e de terapêutica mais complicada ${ }^{(15)}$. Isto também explica o fato de encontrarmos no presente estudo, pacientes com ceceio em idade superior aos 13 anos. Porém, a idade e o tempo de terapia não foram considerados na análise dos dados estatísticos.

O ceceio anterior foi o tipo de distorção mais freqüente na população avaliada, perfazendo um total de $75 \%$ dos casos. Não foram feitas observações e comparações entre os achados 
da pesquisa e os tipos de ceceio encontrados, sendo assim, o tipo de ceceio, lateral ou anterior, não foi considerado na análise dos dados.

Alterações estruturais da cavidade oral, assim como o desequilíbrio das estruturas estomatognáticas alteram significativamente os pontos articulatórios dos fonemas levando a distorções ${ }^{(3,8-10)}$. Das alterações oclusais, encontradas nos pacientes avaliados nesta pesquisa, a mordida aberta anterior foi a de maior ocorrência (50\%), confirmando estudo anterior ${ }^{(9)}$. Esta alteração na emissão do /s/ ocorre devido a uma modificação na posição da língua que se adapta à alteração estrutural para conseguir efetivar a produção desse fonema ${ }^{(7,9,11)}$.

Embora seja possível relacionar a presença de alteração oclusal e ceceio, esse estudo não pode ser considerado conclusivo, pois outros estudos não mostraram essa relação ${ }^{(7,9-11)}$.

A lista de palavras utilizada neste estudo foi desenvolvida, levando-se em conta a existência de palavras que pudessem ser representadas por meio de figuras, facilitando, assim, a sua nomeação. No entanto, houve dificuldade para encontrar todas as palavras e figuras correspondentes a todos os contextos analisados pelas autoras. É importante ressaltar que a lista de palavras poderia ter sido padronizada quantitativamente, contendo o mesmo número de palavras em todos os contextos testados: ataque inicial, ataque medial, coda medial e coda final; em sílabas tônicas e átonas seguidas das vogais [a], [ع], [e], [i], [פ], [o] e [u], facilitando, assim, o processo de análise estatística. O número restrito de participantes da pesquisa pode ter influenciado no resultado estatístico. Caso o estudo fosse realizado com um número maior de dados, os resultados da pesquisa poderiam ser mais expressivos.

Os resultados apontam para o uso da posição inicial do /s/ na sílaba como sugestão de recurso terapêutico, mas é necessário que sejam feitos mais estudos nessa área. Apesar dos resultados obtidos só mostrarem diferenças estatísticas significantes da produção do /s/ nas posições iniciais da sílaba, é interessante relatar para efeito de dado clínico, que foi observada redução discreta e assistemática da ocorrência do ceceio quando /s/ estava nos contextos tônicos da palavra e associado à co-articulação com a vogal / $/$ /.

É importante relatar que, na análise dos dados, foi observado que algumas vogais facilitaram a produção de /s/, embora este dado não tenha sido comprovado estatisticamente: as palavras em que o/s/ co-articulava com a vogal / $/$ /, apresentaram menor índice ceceio, possivelmente por tratar-se de uma vogal posterior.

Salienta-se, portanto, a importância em dar continuidade a este estudo, com um número maior de participantes e um número menor de variáveis. Sugere-se acrescentar alguns critérios de subdivisão em grupos associados e conseqüente diminuição de variáveis, com melhora na padronização do teste de fala (lista de palavras) e a eliminação de outras interferências tais como o tempo de terapia e a diferença de idade entre os pacientes.

\section{CONCLUSÃO}

Após o estudo realizado, verificou-se que o ceceio encontra-se diminuído nas posições ataque inicial, ataque medial em relação às posições de coda medial e coda final. Não foi observada diminuição do ceceio quando tal fonema se apresentava nos contextos silábicos, tônicos ou átonos, ou quando /s/ estava articulado às vogais [a], [ع], [e], [i], [פ], [o] e [u], ou seja, os resultados encontrados não permitem demonstrar associações em relação à tonicidade e à co-articulação com a produção adequada de $/ \mathrm{s} /$. As posições de ataque inicial e medial, porém, parecem ser facilitadoras da produção de [s] e devem, portanto ser consideradas na escolha do recurso e material a ser utilizado em terapia.

\footnotetext{
ABSTRACT

Purpose: This study had the aim to verify and characterize the lisp occurrence, investigating its correlation with the tonicity of the syllable in which the phoneme [s] occurred, either in initial or final position. The study also had the aim to observe whether some of these contexts would facilitate the correct production of the phoneme [s] and would be indicated as a valid therapeutic resource. Methods: From the 54 patients in speech therapy, 16 were selected through records analysis. These subjects (both male and female) had ages ranging from five to 19 years and presented lisp. Data were gathered through a speech test that had the aim to verify the production of the phoneme [s] in 79 words, in initial, middle and final syllable positions, in both stressed and unstressed syllables followed by the vowels [a], [ع], [e], [i], [פ], [o] and [u]. The productions were recorded on audio and video. Statistical analysis used the Minitab software, version 14.0, and the Z Test for comparison of ratio. Results: Twelve patients (75\%) presented frontal lisp and four (25\%), lateral lisp. Reduction of the lisp was not observed in relation to syllable stress or coarticulation with different vowels. The initial and middle syllable positions reduced significantly the lisp, when compared to middle and final coda positions. Conclusion: Syllable stress and coarticulation with different vowels do not modify the incidence of lisp. Initial syllable positions, however, seem to facilitate the production of $[\mathrm{s}]$ and must be considered in the choice of the resource and material to be used in therapy.
}

KEYWORDS: Speech disorders/etiology; Articulation disorders; Phonetics 


\section{REFERÊNCIAS}

1. Wertzner HF. Alterações de fala músculo-esqueléticas: possibilidades de cura. In: Comitê de Motricidade Orofacial da Sociedade Brasileira de Fonoaudiologia. Motricidade Orofacial: como atuam os especialistas. São José dos Campos: Pulso Editorial; 2004. p. 255-7.

2. Zorzi JL. Diferenciando as alterações da fala e da linguagem. In: Marchesan IQ. Fundamentos em fonoaudiologia: aspectos clínicos da motricidade oral. Rio de Janeiro: Ganabara Koogan;1998. p. 59-74.

3. Oliveira JTN, Oliveira ZSB. Desvio fonético X desvio fonológico: algumas considerações. J Bras Fonoaudiol. 2004;5(20):172-6.

4. Wertzner HF. Aquisição da articulação: um estudo em crianças de três a sete anos. Estud Psicol (Campinas). 1994;11(1/2):11-21.

5. Baldi VG, Homem FCB. Caracterização de desvios fonológicos na fala de crianças institucionalizadas de 6 a 7 anos. J Bras Fonoaudiol. 2004;5(18):22-34.

6. Santos GG, Melo PDF, Diniz JMG, Teixeira GPB. A importância do diagnóstico diferencial das alterações de fala: enfoque fonológico. J Bras Fonoaudiol. 2003;4(16):186-92.

7. Pereira MMB, Bianchini EBG, Carvalho GGT, Jardim ZMG. Investigação da ocorrência e caracterização de distorções do /s/ em crianças de 3 a 10 anos. Rev Soc Bras Fonoaudiol. 2003;8(1):10-7.

8. Marchesan IQ. Alterações de fala músculo-esqueléticas: possibilidades de cura. In: Comitê de Motricidade Orofacial da Sociedade Brasileira de Fonoaudiologia. Motricidade Orofacial: como atuam os especialistas. São José dos Campos: Pulso Editorial; 2004. p. 243-9

9. Tomé MC, Farias SR, Araújo SM, Schimitt BE. Ceceio interdental e alterações oclusais em crianças de 03 a 06 anos. Pró-Fono. 2004;16(1):19-30.

10. Rossi KMA, Ávila CRB. Estudo comparativo da produção de fonemas na oclusão normal e maloclusão dental em adolescentes. Pró-Fono. 1999;11(2):77-9.
11. Fonseca RA, Tucci TA, Rodriguez RCL, Gomes ICD, Bianchini EMG. A correlação entre ceceio frontal e o crescimento infantil. Rev Soc Bras Fonoaudiol. 2005;10(4):211-7.

12. Powers MH. Functional disorders of articulation-symptomatology and etiology. In: Travis LE, editor. Handbook of speech pathology and audiology. Englewood Cliffs, N.J.: Prentice Hall; [1971]. p. 837-75.

13. Wertzner HF, Sotelo MB, Amaro L. Analysis of distortions in children with and without phonological disorders. Clinics. 2005;60(2):93-102.

14. Flipsen P Jr, Shriberg L, Weismer G, Karlsson H, McSweeny J. Acoustic characteristics of /s/ in adolescents. J Speech Lang Hear Res. 1999;42(3):663-77.

15. Gonçalves CS, Cielo CA. Desvios fonéticos e fonológicos em paciente adulto: análise de um caso. Fonoaudiol Brasil. 2000;3(4):25-9.

16. Souza RF, Compagnoni MA. Relation between speaking space of the /s/ sound and freeway space in dentate and edentate subjects. Braz Oral Res. 2004;18(4):333-7.

17. Maciel CTV, Leite ICG. Aspectos etiológicos da mordida aberta anterior e suas implicações nas funções orofaciais. Pró-Fono. 2005;17(3):293302.

18. Camara Júnior JM. Estrutura da língua portuguesa. 36a ed. Petrópolis: Vozes; 2004.

19. Lamprecht R. Influência de fatores fonéticos e fonológicos na aquisição das obstruintes sonoras do português. In: Anais do II Encontro Nacional sobre a Aquisição da Linguagem. Porto Alegre: CEAAL/PUCRS; 1991. p.165-84.

20. Britto ATBO. Análise fonético-fonológica do desvozeamento de obstruintes [dissertação]. Belo Horizonte: Programa de Pós-Graduação em Letras. Universidade Federal de Minas Gerais; 2000. 
Anexo 1. Lista de palavras para nomeação

\begin{tabular}{|c|c|c|c|}
\hline \multicolumn{2}{|c|}{ Al- Ataque inicial } & \multicolumn{2}{|c|}{ AM- Ataque medial } \\
\hline Sílaba tônica & Sílaba átona & Sílaba tônica & Sílaba átona \\
\hline Sapo & Sapato & Dinossauro & Pássaro \\
\hline Sal & Sabão & Pulseira & Calça \\
\hline Saia & Sabonete & Travesseiro & Onça \\
\hline Sala & Sanfona & Calcinha & Lingüiça \\
\hline Saco & Salada & Melancia & Urso \\
\hline Seis & Sandália & Vassoura & Osso \\
\hline Sete & Sacola & Girassol & Laço \\
\hline Sino & Saci & & \\
\hline Cinto & Cebola & & \\
\hline Cinco & Cenoura & & \\
\hline Circo & Cinema & & \\
\hline Sopa & Sorvete & & \\
\hline Sol & Sofá & & \\
\hline Suco & Soldado / Sutiã & & \\
\hline \multicolumn{2}{|c|}{ CM - Coda medial } & \multicolumn{2}{|c|}{ CF - Coda final } \\
\hline Sílaba tônica & Sílaba átona & Sílaba tônica & Sílaba átona \\
\hline Máscara & Espada & Nariz & Lápis \\
\hline Pastel & Estrada & Cruz & Tênis \\
\hline Pesca & Escada & Giz & Ônibus \\
\hline Festa & Espuma & Paz & Cartas \\
\hline Testa & Escola & Fez & Talheres \\
\hline Pista & Escova & Nós & Mulheres \\
\hline Disco & Esmalte & & Vinhos \\
\hline Revista & Estrela & & Ninhos \\
\hline Justa & Espelho & & \\
\hline Poste & Disquete & & \\
\hline Ostra & Gustavo & & \\
\hline
\end{tabular}

Anexo 2. Roteiro para fala espontânea

Falar o nome

$\square$ Idade

Data de nascimento

Nome do pai e da mãe

$\square$ Nomes dos irmãos

Para crianças:

$\square$ O que gosta de fazer?(brincar)

- Tem animal de estimação?

$\checkmark$ Nome da escola que estuda e da professora.

- Música e comida preferida.

$\checkmark$ Presente que mais gosta de ganhar.

Para adultos:

$\checkmark$ Se estuda: onde e qual série?

Se trabalha: com o quê?

O que faz nas horas de folga?

Música preferida.

Comida preferida. 